\title{
Full superconducting gap and type-I to type-II superconductivity transition in single crystalline $\mathrm{NbGe}_{2}$
}

\author{
Dongting Zhang, ${ }^{1}$ Tian Le, ${ }^{1}$ Baijiang Lv ${ }^{2}{ }^{2}$ Lichang Yin, ${ }^{1}$ Chufan Chen, ${ }^{1}$ Zhiyong \\ Nie, ${ }^{1}$ Dajun $\mathrm{Su},{ }^{1}$ Huiqiu Yuan, ${ }^{1,3,4,5}$ Zhu-An $\mathrm{Xu},{ }^{2,3,4,5,6}$ and $\mathrm{Xin} \mathrm{Lu}^{1,3,5, *}$ \\ ${ }^{1}$ Center for Correlated Matter and Department of Physics, Zhejiang University, Hangzhou 310058, China \\ ${ }^{2}$ Department of Physics, Zhejiang University, Hangzhou 310027, China \\ ${ }^{3}$ Zhejiang Province Key Laboratory of Quantum Technology and Device, Zhejiang University, Hangzhou 310027, China \\ ${ }^{4}$ State Key Laboratory of Silicon Materials, Zhejiang University, Hangzhou 310027, China \\ ${ }^{5}$ Collaborative Innovation Center of Advanced Microstructures, Nanjing University, Nanjing 210093, China \\ ${ }^{6}$ Zhejiang California International NanoSystems Institute, Zhejiang University, Hangzhou 310058, China
}

(Dated: June 29, 2021)

\begin{abstract}
We report a mechanical point-contact spectroscopy study on the single crystalline $\mathrm{NbGe}_{2}$ with a superconducting transition temperature $T_{\mathrm{c}}=2.0-2.1 \mathrm{~K}$. The differential conductance curves at 0.3 $\mathrm{K}$ can be well fitted by a single gap s-wave Blonder-Tinkham-Klapwijk model and the temperature dependent gap follows a standard Bardeen-Cooper-Schrieffer behavior, yielding $\Delta_{0} \sim 0.32 \mathrm{meV}$ and $2 \Delta_{0} / k_{\mathrm{B}} T_{\mathrm{c}}=3.62$ in the weak coupling limit. In magnetic field, the superconducting gap at $0.3 \mathrm{~K}$ keeps constant up to $H_{c 1} \sim 150$ Oe and gradually decreases until $H_{c 2} \sim 350$ Oe, indicating $\mathrm{NbGe}_{2}$ going through a transition from type-I to type-II (possible type-II/1) superconductor at low temperature.
\end{abstract}

Noncentrosymmetric materials have attracted intensive attention in recent years, whose absence of inversion symmetry in the crystal unit cell can induce an antisymmetric spin-orbit coupling (ASOC) and nontrivial topology of electronic bands ${ }^{1-3}$. If additional mirror or other roto-inversion symmetries are broken in such materials, they form a unique class of topological chiral crystals, and are proposed to host Kramers-Weyl fermions ${ }^{4,5}$. This new type of topological fermions appear at timereversal-invariant momenta with a broad energy range of nontrivial topological bands, and these fermions are connected by Fermi arcs with a large spanning length in the reciprocal space ${ }^{5-7}$. For example, topological bands in the RhSi family have been experimentally observed with characters of chiral crystals, and their helicoid Fermi arcs on surface are claimed to have a Chern number of $\pm 2^{7}$. On the other hand, noncentrosymmetric superconductors can in principle allow an admixture of spinsinglet and -triplet parings ${ }^{1,2,8}$, and serve as a promising platform to realize intrinsic topological superconductors, such as $\mathrm{PbTaSe}_{2}{ }^{9-11}$ and $\mathrm{BiPd}^{12-15}$. Among them, noncentrosymmetric $\mathrm{NbGe}_{2}$ has been reported to have a superconducting transition temperature $T_{c} \sim 2.09 \pm 0.02$ $\mathrm{K}^{16}$ decades ago. Interests on $\mathrm{NbGe}_{2}$ have reemerged due to a recent proposal as a chiral crystal candidate, where its superconductivity and nontrivial topology may be intricately intertwined ${ }^{5}$.

In addition, recent magnetization and specific heat measurements on $\mathrm{NbGe}_{2}$ have confirmed its crossover from type-I to type-II superconductor with decreased temperatures ${ }^{17,18}$, similar to the case of $\mathrm{ZrB}_{12}$ and $\mathrm{LaRhSi}_{3}{ }^{19,20}$. In general, superconductors can be simply classified into type-I and type-II cases, where the Meissner state becomes normal suddenly at $\mathrm{H}>H_{c}$ for type-I superconductors but magnetic field can enter into the sample continuously in the form of quantum flux in type-II superconductors with a transition from the Meiss- ner phase to Shubnikov phase. The Ginzburg-Landau parameter $\kappa=\frac{\lambda}{\xi}$ can be a good indicator, where $\kappa$ for type-I superconductor is smaller than $1 / \sqrt{2}$ and $\kappa$ $>1 / \sqrt{2}$ for type-II case. However, when the $\kappa$ value is close to $1 / \sqrt{2}$, an intermediate mixed (I-M) state can exist between the Meissner state and the mixed state (Shubnikov phase) ${ }^{20-23}$. It has been referred as type-II/1 superconductor in order to be distinguished from the conventional type-II superconductor (type-II/2 superconductor $)^{24}$. For type-II/1 superconductor, a discontinuous increase of the flux density from zero to a certain value $B_{0}$ corresponds to a first-order phase transition from the Meissner state to I-M state at $H_{c 1}{ }^{19,24,25}$. The magnetization usually shows an abrupt decrease from the $4 \pi M=-H$ line at $H_{c 1}$ with a long tail till $M=0$ at $H_{c 2}$, suggesting the I-M and mixed states, respectively ${ }^{17,19,24}$. It is thus desirable to systematically explore the possible topological superconductivity in $\mathrm{NbGe}_{2}$ and its superconducting nature.

In this article, we have applied mechanical pointcontact spectroscopy (MPCS) to investigate the superconducting gap in single crystalline $\mathrm{NbGe}_{2}$. The conductance curves for MPCS on $\mathrm{NbGe}_{2}$ at $0.3 \mathrm{~K}$ can be well fitted by the Blonder-Tinkham-Klapwijk (BTK) model with a single s-wave gap, where the superconducting gap $\Delta$ follows a typical Bardeen-Cooper-Schrieffer (BCS) temperature behavior, yielding $\Delta_{0} \sim 0.32 \mathrm{meV}$ and $2 \Delta_{0} / k_{\mathrm{B}} T_{\mathrm{c}}=3.62$. The Andreev reflection signal in conductance curves keeps the same in magnetic field up to $150 \mathrm{Oe}$, and is gradually suppressed until its upper critical field 350 Oe, consistent with a type-II superconductor at low temperatures.

$\mathrm{NbGe}_{2}$ single crystals were grown by a two-step vapor transport technique, where iodine is the transport agent with stoichiometric amounts of high-purity niobium (99.99\%) and germanium (99.99\%) as described 


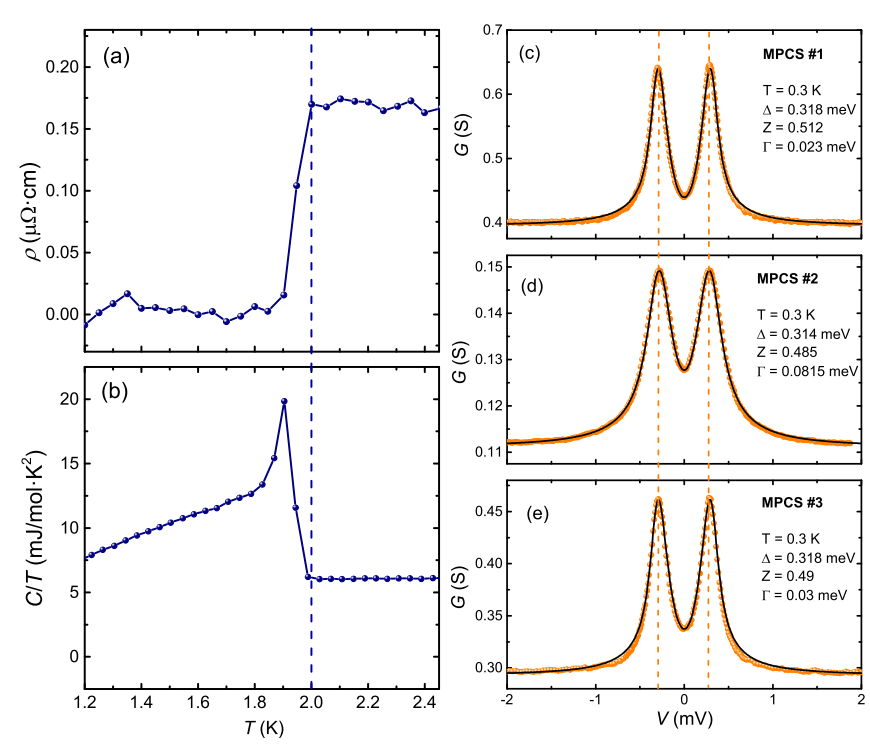

FIG. 1. (color online) (a) and (b) Temperature-dependent electrical resistivity $\rho$, and specific heat $\mathrm{C} / \mathrm{T}$ of $\mathrm{NbGe}_{2}$, respectively; (c)-(e) A representative set of point-contact conductance curves on $\mathrm{NbGe}_{2}$ at $0.3 \mathrm{~K}$ from MPCS, in comparison with a single gap s-wave BTK fitting (black lines).

elsewhere ${ }^{17}$. The electrical resistivity and specific-heat of $\mathrm{NbGe}_{2}$ were measured by a Physical Property Measurement System (PPMS) from Quantum Design with a Helium 3 insert cooling down to $0.4 \mathrm{~K}$. Both the specificheat jump and resistive drop at $T_{\mathrm{c}} \sim 2 \mathrm{~K}$ support the high quality of the $\mathrm{NbGe}_{2}$ crystals, as shown in Fig. 1(a) and (b). The sharp specific jump in Fig. 1(b) is probably due to a remnant field in PPMS causing a first-order transition, consistent with the reported specific-heat behavior in field ${ }^{17,18}$. Mechanical PCS in a needle-anvil type was employed to study the superconducting gap of $\mathrm{NbGe}_{2}$, where a sharp gold tip prepared by the electrochemical etching was gently engaged on the crystal surface by piezoelectric-controlled nanopositioners. For the point-contact, its differential conductance curves as a function of bias voltage, $G(V)$, were measured in a quasifour-probe configuration by the conventional lock-in technique, where the experimental details can be referred to 26 and 27. The ac susceptibility of $\mathrm{NbGe}_{2}$ was measured in coils by generating an ac field of $1337 \mathrm{~Hz}$ in frequency and 0.8 Oe in amplitude, and both the dc and ac fields are perpendicular to the polished sample surface along the $c$ axis. Oxford cryostat with a He 3 insert was used for MPCS and ac susceptibility measurements to cool the sample down to $0.3 \mathrm{~K}$ and to apply magnetic field up to 1000 Oe.

Figure 1(c)-(e) show a representative set of differential conductance curves $\mathrm{G}(\mathrm{V})$ at $0.3 \mathrm{~K}$ for three different contacts on $\mathrm{NbGe}_{2}$. All the conductance curves have a common double-peak structure and can be well fitted by a single-gap s-wave BTK model, suggesting a full superconducting gap in $\mathrm{NbGe}_{2}$. The obtained gap $\Delta \sim 0.316$ $\mathrm{meV}$ at $0.3 \mathrm{~K}$ and the smearing factor $\Gamma$ varies between
0.02 and $0.08 \mathrm{meV}$, signaling a small scattering rate and thus a clean interface for our point-contacts. A slight deviation of the experimental conductance curve from the BTK fitting can be noticed for Fig. 1(e) at high bias voltages but should not undermine our analysis ${ }^{28}$. The temperature evolution of point-contact conductance curves are shown in Fig. 2(a) from $0.3 \mathrm{~K}$ to $2.1 \mathrm{~K}$, where the double peaks gradually shift to zero-bias with increased temperatures and the $\mathrm{G}(\mathrm{V})$ curve becomes flat at $2.1 \mathrm{~K}$ for $\mathrm{NbGe}_{2}$ in the normal state. If we track the temperature dependent zero-bias conductance (ZBC) of MPCS on $\mathrm{NbGe}_{2}$ as in Fig. 2(b), a kink at $2.05 \mathrm{~K}$ in the $\mathrm{ZBC}$ curve indicates the disappearance of Andreev reflection and that the $\mathrm{NbGe}_{2}$ crystal transforms from superconducting into normal state, consistent with the resistivity and specific heat data. For all the measured contacts on $\mathrm{NbGe}_{2}$, they have a narrow range of superconducting transition temperatures $T_{\mathrm{c}}=2.0-2.1 \mathrm{~K}$. The extracted superconducting gap values $\Delta$ from the BTK fitting are plotted in Fig. 2(c) as a function of the reduced temperature $T / T_{c}$ and they follow the typical BCS temperature behavior, yielding $\Delta_{0} \sim 0.32 \mathrm{meV}$ and $2 \Delta_{0} / k_{\mathrm{B}} T_{\mathrm{c}}=3.62$ in the weak-coupling regime, close to the reported value 3.528 from the specific heat ${ }^{18}$. We note that the tunneling barrier parameter $\mathrm{Z}$ keeps almost constant while the ratio between the smearing parameter $\Gamma$ and superconducting gap $\Delta, \Gamma / \Delta$, sharply increases close to $T_{\mathrm{c}}$ as in the inset of Fig. 2(c) due to the enhanced pair-breaking effect $^{29}$.

The point-contact conductance curves at $0.3 \mathrm{~K}$ shows an interesting behavior in magnetic field as in Fig. 3(a): They do not change with magnetic field at all up to 150 Oe, characteristic of the Meissner state in field, and the conductance peak intensity is dramatically suppressed by field above $150 \mathrm{Oe}$, in reminiscence of our previous MPCS observations on the type-I superconductor $\mathrm{PdTe}_{2}{ }^{30}$. However, in contrast to the case of $\mathrm{PdTe}_{2}$, the peak positions shift to zero-bias voltage and the doublepeak distance gradually decreases, similar to the behavior of a conventional type-II superconductor in its mixed state instead ${ }^{30,31}$. The Andreev reflection signal is totally suppressed and the curves become flat above its upper critical field $H_{c 2} \sim 350$ Oe. The gradual decrease of double-peak distance supports $\mathrm{NbGe}_{2}$ as a type-II superconductor at low temperature $0.3 \mathrm{~K}$, implying a transition from type-I to type-II superconductivity with decreased temperature as reported for $\mathrm{NbGe}_{2}{ }^{17,18}$. The ZBC curves as a function of field for three different contacts are plotted in Fig. 3(b) with a consistent manner, where their conductance values keep constant up to 150 Oe and decrease until its upper critical field $H_{c 2} \sim 350$ Oe. The ZBC behavior is similar to the results on typeI superconductors $\mathrm{Al}$, $\mathrm{Sn}$ (as shown in the Supplemental Material ${ }^{32}$ ) and $\mathrm{PdTe}_{2}{ }^{30}$, however, we note the field range for the conductance change of $\mathrm{NbGe}_{2}(\sim 200 \mathrm{Oe})$ is much broader in comparison.

In order to argue against the scenario that the smooth decrease of the conductance peak intensity above 150 

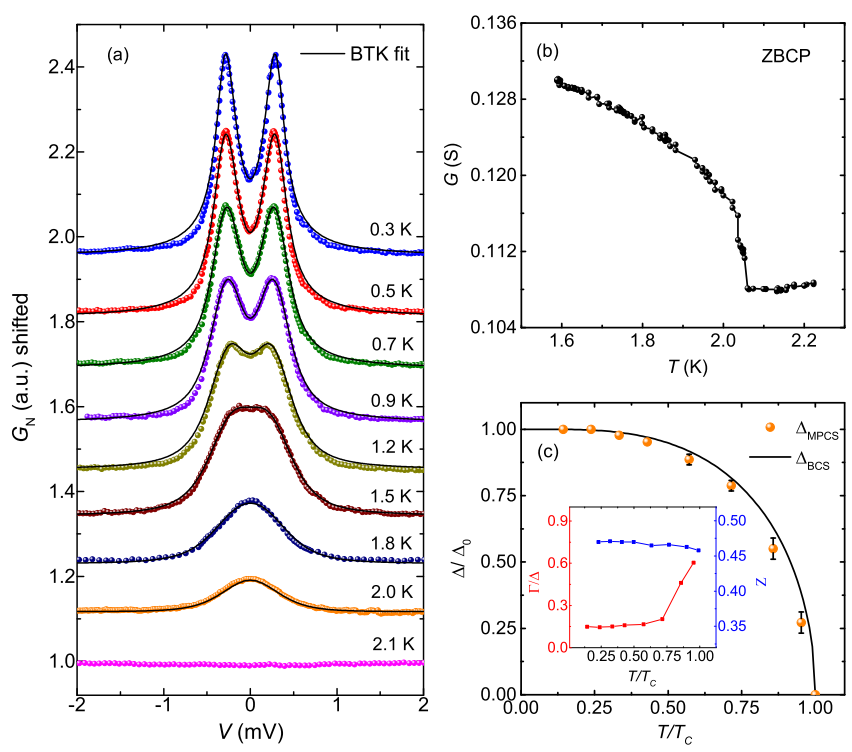

FIG. 2. (color online) (a) Temperature evolution of the normalized differential conductance curves $G_{\mathrm{N}}(\mathrm{V})$ from 0.3 to $2.1 \mathrm{~K}$ for MPCS on $\mathrm{NbGe}_{2}$, in comparison with a single gap s-wave BTK fitting (black lines). The curves are shifted vertically for clarity. (b) The MPCS zero-bias conductance as a function of temperature with a kink at $T_{c} \sim 2-2.1 \mathrm{~K}$. (c) Temperature dependence of the extracted superconducting gap $\Delta$ from the single-gap BTK fitting (solid circle) in comparison with the standard BCS temperature curve (black line). The inset shows the fitting parameters $\mathrm{Z}$ and $\Gamma / \Delta$ as a function of temperature.

Oe originates from the intermediate state for a typeI superconductor as in $\mathrm{PdTe}_{2}{ }^{30}$, we have tried to analyze $\mathrm{NbGe}_{2}$ conductance data at 200 Oe with the same two-component BTK model $G(V)=\omega G_{\text {Normal }}+(1-$ $\omega) G_{\mathrm{SC}}(V)$, which works well for soft PCS on type-I superconductors $\mathrm{PdTe}_{2}{ }^{30}$ and $\mathrm{Al}$ (Refer to FigS1 in the Supplemental Material ${ }^{32}$ ). In the intermediate state of a type-I superconductor, there exists a phase separation between the Meissner superconducting state and normal state due to a large demagnetization factor. The weighting parameter $\omega$ for the normal state is proportional to its volume fraction in the contact area and the conductance $G_{\mathrm{SC}}(V)$ from the superconducting part should gave the same shape as in the zero-field case, where the gap $\Delta, \Gamma$ and $\mathrm{Z}$ are constant in the Meissner state. In $\mathrm{PdTe}_{2}$, the experimental curves $G(V)$ can be well fitted by only changing the spectra weight $\omega$ for different fields near $H_{c}$, implying an intermediate state for the typeI superconductor ${ }^{30}$. However, the same procedure fails when fitting the conductance curve of $\mathrm{NbGe}_{2}$ at $200 \mathrm{Oe}$, with respect to both the peak intensity and position, as shown in Fig. 3(c) by the dashed lines. In comparison, the conductance curve at 200 Oe can be well fitted by the BTK model with a reduced gap value, suggesting $\mathrm{NbGe}_{2}$ in the type-II-like mixed state instead. In the pure Meissner state of $\mathrm{NbGe}_{2}$ below $150 \mathrm{Oe}, \omega$ is simply zero and thus the total conductance $\mathrm{G}(\mathrm{V})$ would not change. Our mechanical point-contact results in field would thus fa-
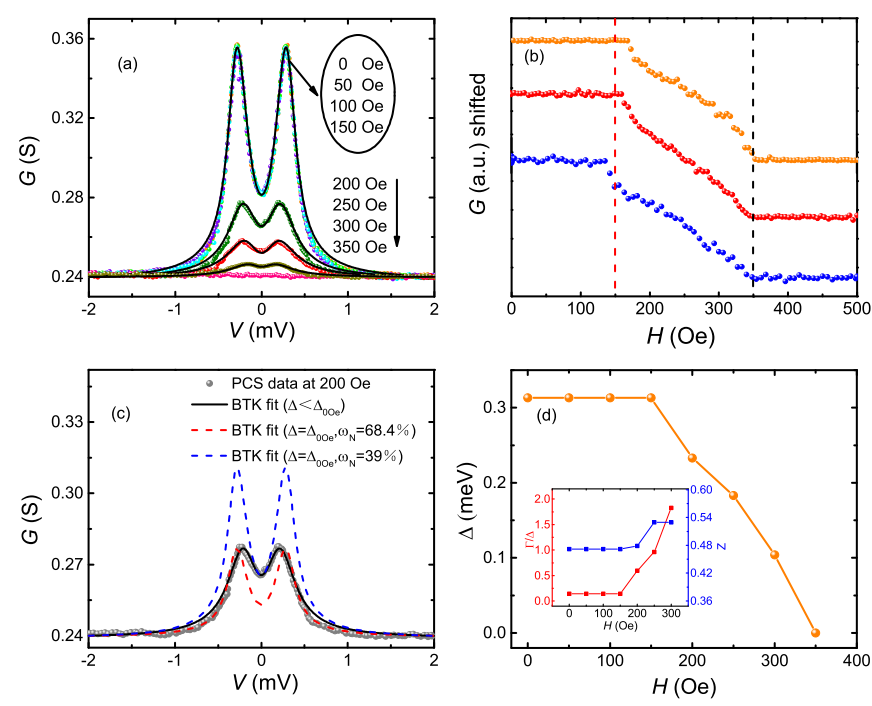

FIG. 3. (Color online) (a) Field evolution of the differential conductance curves $G(V)$ from zero to 350 Oe for MPCS on $\mathrm{NbGe}_{2}$ at $0.3 \mathrm{~K}$, in comparison with the single gap s-wave BTK fitting curves (black lines). (b) The zero bias conductance of three different contacts on on $\mathrm{NbGe}_{2}$ at $0.3 \mathrm{~K}$ as a function of field. The upper critical field is marked by a black dashed line while the red dashed line marks the lower critical field $H_{\mathrm{c} 1}$. (c) Different Fitting curves with the single-gap or two-component BTK model by varying $\omega$ (68.4\% and $39 \%)$ in comparison with the differential conductance curve at 200 Oe. (d) Field dependence of the superconducting gap $\Delta$ extracted from the single-gap BTK fitting. The inset shows the fitting parameters $\mathrm{Z}$ and $\Gamma / \Delta$ as a function of field.

vor $\mathrm{NbGe}_{2}$ as a type-II rather than type-I superconductor at $0.3 \mathrm{~K}$. In both the Meissner state below 150 Oe and mixed state between 150 and 350 Oe, the conductance curves for point-contact on $\mathrm{NbGe}_{2}$ can be well fitted by the one-gap s-wave BTK model as in Fig. 3(a) and the extracted parameters are shown in Fig. 3(d) and its inset, where the superconducting gap keeps constant below 150 Oe and decreases to zero at $H_{c 2}$ while the $\Gamma / \Delta$ dramatically increases above 150 Oe with flux entering the $\mathrm{NbGe}_{2}$ crystal.

We have measured the field-dependent ac susceptibility of $\mathrm{NbGe}_{2}$ at different temperatures with its imaginary part $\chi^{\prime \prime}(H)$ and real part $\chi^{\prime}(H)$ shown in Fig. 4(a) and (b), respectively, where $\chi^{\prime}$ indicates the shielding ability of superconductor and $\chi^{\prime \prime}$ reflects the magnetic irreversibility $^{33}$. A differential paramagnetic effect is observed at $2 \mathrm{~K}$, where the real part $\chi^{\prime}$ shows a noticeable positive spike ${ }^{34}$. It should originate from the rapid transition from the $\mathrm{M}=-\mathrm{H}$ to $\mathrm{M}=0$ state, which is common in type-I and type-II/1 superconductors ${ }^{20,35-38}$. With reduced temperatures, the transition range of $\chi^{\prime}$ from the minimum (Meissner state) to zero (normal state) becomes larger, while the peak in $\chi^{\prime \prime}(\mathrm{H})$ shifts to higher field and gets broader, showing an increased critical field. The absence of the positive spike in the real part $\chi^{\prime}$ at low temperatures is obviously different from the type-I superconductor behavior ${ }^{36,39}$ (as demonstrated for both 

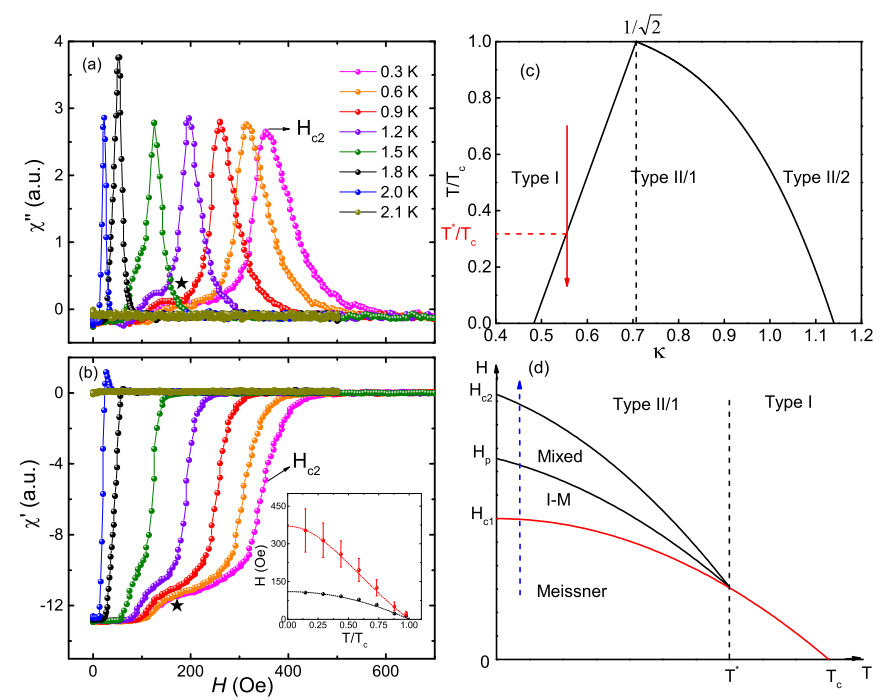

FIG. 4. (color online) (a) and (b) The field dependent ac susceptibility of $\mathrm{NbGe}_{2}$ at different temperatures with its imaginary and real parts, respectively. Black stars mark the small plateau in $\chi^{\prime \prime}(H)$ and a small step in $\chi^{\prime}(H)$ in the field region of 100 - 200 Oe. The upper critical field at $0.3 \mathrm{~K}$ is marked by black arrows at the peak of $\chi^{\prime \prime}(H)$ and in $\chi^{\prime}(H)$. In the inset of (b), the peak of $\chi^{\prime \prime}(H)$ is defined as upper critical field $H_{c 2}$ (red dot) with its full width at half maxima (FWHM) taken as the error bar (vertical line). The lower critical field $H_{c 1}$ is defined where $\chi^{\prime}$ starts to increase from the minimum (black dot). $H_{c 2}$ and $H_{c 1}$ follows different temperature-dependent GL formula (dashed lines). (c) Phase diagram of superconductors with type I, type-II/1 and type-II/2 superconductor regions in the $\mathrm{T} / \mathrm{T}_{c^{-}} \kappa$ plane. The red line shows a transition from type-I to type-II/1 superconductor at $T^{*}$ with decreased temperatures. (d) H-T phase diagram for $\kappa$ in the range of $\sim 0.5<\kappa<1 / \sqrt{2}$, where it transforms from type-I to type-II/ 1 superconductor at $T^{*}$. Solid lines show the phase boundaries between Meissner, intermediate-mixed, mixed and normal states, where the first-order phase transition is in red color and second-order phase transitions in black color. (c) and (d) are adapted from Ref. [20].

$\mathrm{Al}$ and Sn in FigS4 of the Supplemental Material $\left.{ }^{32}\right)$. At the lowest temperature $0.3 \mathrm{~K}$, the $\chi^{\prime}(\mathrm{H})$ keeps constant below 110 Oe with a shielding effect in the Meissner state and continuously increases above 110 Oe till 350 Oe, supporting a type-II superconductivity in $\mathrm{NbGe}_{2}$ at $0.3 \mathrm{~K}$. If we define the peak position in $\chi^{\prime \prime}(H)$ as the upper critical field $H_{c 2}$ and the deviation field from the Meissner state as the $H_{c 1}$ at $0.3 \mathrm{~K}$, they would be 353 and 106 Oe, respectively, consistent with the values from point-contact spectroscopy. The temperature dependent $H_{c 1}(T)$ and $H_{c 2}(T)$ are extracted in the same manner as $0.3 \mathrm{~K}$ and shown in the inset of Fig. 4(b), which follow the GL formula $H_{c 1}(T)=H_{c 1}(0)\left[1-\left(\frac{T}{T_{c}}\right)^{2}\right]$ and $H_{c 2}(T)$ $=H_{c 2}(0) \frac{\left[1-\left(\frac{T}{T_{c}}\right)^{2}\right]}{\left[1+\left(\frac{T}{T_{c}}\right)^{2}\right]}$, respectively.

Our MPCS and ac susceptibility measurements have established $\mathrm{NbGe}_{2}$ as a type-I $\mathrm{SC}$ at high temperature and type-II SC at $0.3 \mathrm{~K}$. Meanwhile, a transition from
type-I to type-II superconductor with decreased temperature has also been confirmed by the specific heat and isothermal magnetization measurements on $\mathrm{NbGe}_{2}{ }^{17,18}$. We speculate that $\mathrm{NbGe}_{2}$ might be an intrinsic typeII/1 superconductor as in Fig. 4(c). As stated earlier, besides the type-I and conventional type-II superconductors classified by the Ginzburg-Landau (GL) parameter $\kappa$, there exists a new branch of type-II/1 superconductor distinct from the conventional type-II superconductor (type-II/2 superconductor), when its $\kappa$ is in the range of $\sim 0.5<\kappa<1 / \sqrt{2}$. In such a case, a transition from type-I to type-II/1 can occur for the superconductor at $T^{*}$ with decreased temperatures as illustrated in Fig. $4(\mathrm{c})^{24}$. Moreover, the superconductor below $T^{*}$ would go through several phases in field, such as Meissner state, intermediate-mixed state, and mixed state before entering into the normal state as in Fig. 4(d). We note that the $\kappa$ value estimated in Ref. 17 is only 0.12 , much smaller than the phase boundary of 0.5 or $1 / \sqrt{2}$. On the other hand, the Maki parameter $\kappa_{1}$ is defined as $\kappa_{1}(T)=\frac{1}{\sqrt{2}} \frac{H_{c 2}(T)}{H_{c}(T)}$, and $\kappa_{1}=\kappa$ with the limit $\mathrm{T} \rightarrow T_{c}$. In general, the condition $\kappa_{1}\left(T^{*}\right)=\frac{1}{\sqrt{2}}$ determines the transition temperature $T^{*}$ from type-I to type-II SC $\mathrm{SC}^{24}$. We can infer that the $T^{*}=1.4 \mathrm{~K}$ where $H_{c}(T)$ deviates from $H_{c 2}(T)^{19}$, and $\kappa_{1}(0 K) \simeq 1.14$ with the critical field $H_{c 2}(0)=360$ Oe and $H_{c}(0)=223$ Oe from the $\mathrm{H}$ - $\mathrm{T}$ phase diagram in Ref. 17. If we assume a linear temperature dependence of $\kappa_{1}(\mathrm{~T})$, the obtained GL parameter $\kappa \equiv$ $\kappa_{1}\left(T_{c}\right)=0.52$ for $\mathrm{NbGe}_{2}$ is right in the region of $\sim 0.5<$ $\kappa<1 / \sqrt{2}$, consistent with its type-II/1 superconducting behaviors. Above the low critical field $H_{c 1} \sim 150 \mathrm{Oe}$, the ac susceptibility shows a small step in $\chi^{\prime}(H)$ and a plateau in $\chi^{\prime \prime}(H)$ in Fig. 4(b), which might be a signal of an abrupt entry of magnetic flux with a discontinuous density in type-II/1 superconductor. However, more microscopic studies are required to further confirm the existence of an intermediate-mixed state and the typeII/1 nature of $\mathrm{NbGe}_{2}$.

In conclusion, we have observed a single full s-wave gap $\Delta$ for the chiral crystal candidate $\mathrm{NbGe}_{2}$ from pointcontact spectroscopy and the gap $\Delta$ follows a typical BCS temperature behavior, yielding $\Delta_{0} \sim 0.32 \mathrm{meV}$ with $2 \Delta_{0} / k_{\mathrm{B}} T_{\mathrm{c}}=3.62$ in the weak coupling limit. The differential conductance curves for $\mathrm{NbGe}_{2}$ in magnetic field show a switch from the Meissner state below 150 Oe to a mixed state between 150 and 350 Oe at $0.3 \mathrm{~K}$, characteristic of a possible type-II/1 superconductor at low temperatures.

We are grateful for valuable discussions with Q.H. Chen, C. Cao and Y. Liu. Work at Zhejiang University was supported by the National Key Research \& Development Program of China (Grant No. 2016YFA0300402 and 2017YFA0303101) and the National Natural Science Foundation of China (Grant No. 11674279, No. 11774305, No. 11604291, No. 11374257 and No. 11974306). X.L. would like to acknowledge support from the Zhejiang Provincial Natural Science Foundation of China (LR18A04001). 
* Corresponding author: xinluphy@zju.edu.cn

1 Sungkit Yip, "Noncentrosymmetric superconductors," Annual Review of Condensed Matter Physics 5, 15-33 (2013)

2 M Smidman, M B Salamon, $H$ Q Yuan, and D F Agterberg, "Superconductivity and spin-orbit coupling in non-centrosymmetric materials: a review," Reports on Progress in Physics 80, 036501 (2017).

3 Peiran Zhang, Huiqiu Yuan, and Chao Cao, "Electronphonon coupling and nontrivial band topology in noncentrosymmetric superconductors LaNiSi, LaPtSi, and LaPtGe," Phys. Rev. B 101, 245145 (2020).

4 Howard D. Flack, "Chiral and Achiral Crystal Structures," Helvetica Chimica Acta 86, 905-921 (2003).

5 Guoqing Chang, Benjamin J. Wieder, Frank Schindler, Daniel S. Sanchez, Ilya Belopolski, Shin-Ming Huang, Bahadur Singh, Di Wu, Tay-Rong Chang, Titus Neupert, Su-Yang $\mathrm{Xu}$, Hsin Lin, and M. ZahidHasa, "Topological quantum properties of chiral crystals," Nature Materials 17, 978-985 (2018).

6 Chandra Shekhar, "Chirality meets topology," Nature Materials 17, 953-954 (2018).

7 Daniel S. Sanchez, Ilya Belopolski, Tyler A. Cochran, Xitong Xu, Jia-Xin Yin, Guoqing Chang, Weiwei Xie, Kaustuv Manna, Vicky Süß, Cheng-Yi Huang, Nasser Alidoust, Daniel Multer, Songtian S. Zhang, , Nana Shumiya, Xirui Wang, Guang-Qiang Wang, Tay-Rong Chang, Claudia Felser, Su-Yang Xu, Shuang Jia, Hsin Lin, and M. Zahid Hasan, "Topological chiral crystals with helicoid-arc quantum states," Nature 567, 500-505 (2019).

8 S. S. Saxena and P. Monthoux, "Symmetry not required," Nature 427, 799 (2004).

9 Mazhar N. Ali, Quinn D. Gibson, T. Klimczuk, and R. J. Cava, "Noncentrosymmetric superconductor with a bulk three-dimensional Dirac cone gapped by strong spin-orbit coupling," Phys. Rev. B 89, 020505 (2014).

10 Syu-You Guan, Peng-Jen Chen, Ming-Wen Chu, Raman Sankar, Fangcheng Chou, Horng-Tay Jeng, Chia-Seng Chang, and Tien-Ming Chuang, "Superconducting topological surface states in the noncentrosymmetric bulk superconductor $\mathrm{PbTaSe}_{2}, "$ Science Advances 2, 1600894 (2016).

11 Tian Le, Yue Sun, Hui-Ke Jin, Liqiang Che, Lichang Yin, Jie Li, Guiming Pang, Chunqiang Xu, Lingxiao Zhao, Shunichiro Kittaka, Toshiro Sakakibara, Kazushige Machida, Raman Sankar, Huiqiu Yuan, Genfu Chen, Xiaofeng Xu, Shiyan Li, Yi Zhou, and XinLu, "Evidence for nematic superconductivity of topological surface states in $\mathrm{PbTaSe}_{2}$," Science Bulletin 65, 1349-1355 (2020).

12 Mintu Mondal, Bhanu Joshi, Sanjeev Kumar, Anand Kamlapure, Somesh Chandra Ganguli, Arumugam Thamizhavel, Sudhansu S. Mandal, Srinivasan Ramakrishnan, and Pratap Raychaudhuri, "Andreev bound state and multiple energy gaps in the noncentrosymmetric superconductor BiPd," Phys. Rev. B 86, 094520 (2012).

13 Madhab Neupane, Nasser Alidoust, M. Mofazzel Hosen, Jian-Xin Zhu, Klauss Dimitri, Su-Yang Xu, Nagendra Dhakal, Raman Sankar, Ilya Belopolski, Daniel S. Sanchez, Tay-Rong Chang, Horng-Tay Jeng, Koji Miyamoto, Taichi Okuda, Hsin Lin, Arun Bansil, Dariusz Kaczorowski, Fangcheng Chou, M. Zahid Hasan, and Tomasz Durakiewicz, "Observation of the spin-polarized surface state in a noncentrosymmetric superconductor BiPd," Nature communication 7, 13315 (2016).

${ }^{4}$ Mojammel A. Khan, D. E. Graf, I. Vekhter, D. A. Browne, J. F. DiTusa, W. Adam Phelan, and D. P. Young, "Quantum oscillations and a nontrivial berry phase in the noncentrosymmetric topological superconductor candidate BiPd," Phys. Rev. B 99, 020507 (2019).

15 Xiaoying $\mathrm{Xu}$, Yufan Li, and C. L. Chien, "Spintriplet pairing state evidenced by half-quantum flux in a noncentrosymmetric superconductor," Phys. Rev. Lett. 124, 167001 (2020).

16 J. P. Pemeika and A. S. Cooper, "Superconductivity of single-crystal $\mathrm{NbGe}_{2}$," Journal of the Less-Cordon Metals 62, 211-213 (1978).

17 Baijiang Lv, Miaocong Li, Jia Chen, Yusen Yang, Siqi Wu, Lei Qiao, Feihong Guan, Hui Xing, Qian Tao, Guang-Han Cao, and Zhu-An Xu, "TypeI superconductivity in noncentrosymmetric $\mathrm{NbGe}_{2}$," Phys. Rev. B 102, 064507 (2020).

18 Eve Emmanouilidou, Sougata Mardanya, Jie Xing, P. V. Sreenivasa Reddy, Amit Agarwal, Tay-Rong Chang, and $\mathrm{Ni} \mathrm{Ni}$, "Fermiology and type-I superconductivity in the chiral superconductor $\mathrm{NbGe}_{2}$ with Kramers-Weyl fermions," Phys. Rev. B 102, 235144 (2020).

19 Yuxing Wang, Rolf Lortz, Yuriy Paderno, Vladimir Filippov, Satoko Abe, Ulrich Tutsch, and Alain Junod, "Specific heat and magnetization of a $\mathrm{ZrB}_{12}$ single crystal: Characterization of a type-II/1 superconductor," Phys. Rev. B 72, 024548 (2005).

20 Noriaki Kimura, Noriyuki Kabeya, Kohsuke Saitoh, Kazunori Satoh, Hajime Ogi, Kohki Ohsaki, and Haruyoshi Aoki, "Type II/1 superconductivity with Extremely High $\mathrm{H}_{\mathrm{c} 3}$ in Noncentrosymmetric $\mathrm{LaRhSi}_{3}$," J. Phys. Soc. Jpn. 85, 024715 (2016).

21 S. Mühlbauer, C. Pfleiderer, P. Böni, M. Laver, E. M. Forgan, D. Fort, U. Keiderling, and G. Behr, "Morphology of the superconducting vortex lattice in ultrapure niobium," Phys. Rev. Lett. 102, 136408 (2009).

22 T. Reimann, S. Mühlbauer, M. Schulz, B. Betz, A. Kaestner, V. Pipich, P. Böni, and C. Grünzweig, "Visualizing the morphology of vortex lattice domains in a bulk type-II superconductor," Nature Communications 6, 8813 (2015).

23 A. Backs, M. Schulz, V. Pipich, M. Kleinhans, P. Böni, and S. Mühlbauer, "Universal behavior of the intermediate mixed state domain formation in superconducting niobium," Phys. Rev. B 100, 064503 (2019).

24 J. Auer and H. Ullmaier, "Magnetic behavior of TypeII Superconductors with Small Ginzburg-Landau Parameters," Phys. Rev. B 7, 136-145 (1973).

25 L. Leplae, V. Srinivasan, and H. Umezawa, "Theory for type-II/1 superconductors." Lett. Nuovo Cimento 9, 711-715 (1974).

26 M. Tortello, W. K. Park, C. O. Ascencio, P. Saraf, and L. H. Greene, "Design and construction of a pointcontact spectroscopy rig with lateral scanning capability," Review of Scientific Instruments 87, 063903 (2016).

27 Shekhar Dasa and Goutam Sheet, "A modular point contact spectroscopy probe for sub-Kelvin applications," Review of Scientific Instruments 90, 103903 (2019).

28 Goutam Sheet, S. Mukhopadhyay, and P. Raychaudhuri, "Role of critical current on the point-contact andreev re- 
flection spectra between a normal metal and a superconductor," Phys. Rev. B 69, 134507 (2004).

29 D Daghero and R S Gonnelli, "Probing multiband superconductivity by point-contact spectroscopy," Superconductor Science and Technology 23, 043001 (2010).

30 Tian Le, Lichang Yin, Zili Feng, Qi Huang, Liqiang Che, Jie Li, Youguo Shi, and Xin Lu, "Single full gap with mixed type-I and type-II superconductivity on surface of the type-II dirac semimetal $\mathrm{PdTe}_{2}$ by point-contact spectroscopy," Phys. Rev. B 99, 180504 (2019).

31 Qi Huang, Tian Le, LiQiang Che, Lichang Yin, Jie Li, Jinhu Yang, Minghu Fang, and Xin Lu, "A single full gap in noncentrosymmetric superconductor $\mathrm{Re}_{6} \mathrm{Hf}$ by point-contact spectroscopy," Materials Research Express 6, 016001 (2018).

32 See Supplemental Material for more discussions about point-contact spectroscopy and ac susceptibility measurements on some classical type-I superconductors such as Al and Sn crystals.

33 Fedor Gömöry, "Characterization of high-temperature superconductors by AC susceptibility measurements," Superconductor Science and Technology 10, 523-542 (1997).

${ }^{34}$ Robert A. Hein and Raymond L. Falge, "Dif- ferential paramagnetic effect in superconductors," Phys. Rev. 123, 407-415 (1961).

35 T. T. M. Palstra, G. Lu, A. A. Menovsky, G. J. Nieuwenhuys, P. H. Kes, and J. A. Mydosh, "Superconductivity in the ternary rare-earth (Y, $\mathrm{La}$, and $\mathrm{Lu})$ compounds $\mathrm{RPd}_{2} \mathrm{Si}_{2}$ and $\mathrm{RRh}_{2} \mathrm{Si}_{2}$," Phys. Rev. B 34, 4566-4570 (1986).

36 Liang L. Zhao, Stefan Lausberg, H. Kim, M. A. Tanatar, Manuel Brando, R. Prozorov, and E. Morosan, "Type-I superconductivity in $\mathrm{YbSb}_{2}$ single crystals," Phys. Rev. B 85, 214526 (2012).

37 H. Leng, C. Paulsen, Y. K. Huang, and A. de Visser, "Type-I superconductivity in the dirac semimetal $\mathrm{PdTe}_{2}$," Phys. Rev. B 96, 220506 (2017).

38 Darren C. Peets, Erjian Cheng, Tianping Ying, Markus Kriener, Xiaoping Shen, Shiyan Li, and Donglai Feng, "Type-I superconductivity in $\mathrm{Re}_{6} \mathrm{Al}$," Phys. Rev. B 99, 144519 (2019).

39 Shingo Yonezawa and Yoshiteru Maeno, "Type-I superconductivity of the layered silver oxide $\mathrm{Ag}_{5} \mathrm{~Pb}_{2} \mathrm{O}_{6}$," Phys. Rev. B 72, 180504 (2005). 